\title{
Traumatic abdominal wall hernias in children: A case for early exploration
}

\author{
Niloufar Hafezi MD ${ }^{\mathrm{a}}$, Jodi L. Raymond MPH ${ }^{\mathrm{b}}$, Erin D. Mayo CPNP ${ }^{\mathrm{b}}$, Thomas M. Rouse MD ${ }^{\mathrm{a}, \mathrm{b}}$, \\ Deborah F. Billmire MD ${ }^{\mathrm{a}, \mathrm{b}}$, Brian W. Gray $\mathrm{MD}^{\mathrm{a}, \mathrm{b}}$ \\ ${ }^{a}$ Department of Surgery, Division of Pediatric Surgery, Indiana University School of Medicine, \\ 705 Riley Hospital Drive, Suite 2500, Indianapolis, IN 46202, USA \\ ${ }^{\mathrm{b}}$ Riley Trauma Program, Riley Hospital for Children, Indiana University Health, \\ 705 Riley Hospital Drive, Suite 1925, Indianapolis, IN 46202, USA
}

Declarations of interest: none

\section{Corresponding Author Address:}

Brian W. Gray, MD, FACS, FAAP

Assistant Professor of Pediatric Surgery

Riley Hospital for Children

Indiana University School of Medicine

705 Riley Hospital Dr.

RI 2500

Indianapolis, IN 46202

Email: graybw@iupui.edu

Phone: $317-437-2506$

Fax: 317-274-4491

Abbreviated Title: Traumatic abdominal wall hernias in children

This is the author's manuscript of the article published in final edited form as:

Hafezi, N., Raymond, J. L., Mayo, E. D., Rouse, T. M., Billmire, D. F., \& Gray, B. W. (2020). Traumatic abdominal wall hernias in children: A case for early exploration. Journal of Pediatric Surgery. https://doi.org/10.1016/j.jpedsurg.2020.06.046 


\begin{abstract}
:
Purpose: Traumatic abdominal wall hernia (TAWH) is a rare consequence of blunt abdominal trauma (BAT). We examined a series of patients suffering TAWH to evaluate its frequency, rate of associated concurrent intrabdominal injuries (CAI) and correlation with CT, management and outcomes.

Methods: A Level 1 pediatric trauma center trauma registry was queried for children under 18 suffering TAWH from BAT between 2009-2019.

Results: 9370 patients were admitted after BAT. TAWH was observed in 11 children, at incidence $0.1 \%$. Eight children (73\%) were male, at mean age 10 years, and mean ISS of 16 . Six cases $(55 \%)$ were due to MVC, three $(27 \%)$ impaled by a handlebar or pole, and two $(18 \%)$ dragged under large machinery. Seven (64\%) had a CAI requiring operative or interventional management. Patients with CAI were similar to those without other injury, with $20 \%$ and $50 \%$ CT scan sensitivity and specificity for detection of associated injury, respectively. Five patients had immediate hernia repair with laparotomy for repair of intrabdominal injury, three had delayed repair, two have asymptomatic unrepaired TAWH, and one resolved spontaneously. Conclusions: Children with TAWH have high rates of CAI requiring operative repair. CT scans have low sensitivity and specificity for detecting associated injuries. A high suspicion of injury and low threshold for exploration must be maintained in TAWH cases.

Key Words: Traumatic abdominal wall hernia, pediatric, traumatic hernia, abdominal disruption

Level of evidence: IV

Abbreviations: Traumatic abdominal wall hernia (TAWH); blunt abdominal trauma (BAT); concurrent intrabdominal injury (CAI)
\end{abstract}




\section{INTRODUCTION:}

Traumatic abdominal wall hernia (TAWH) is a sequela of blunt trauma to the abdominal wall causing deep muscle or fascial disruption without sufficient force to penetrate the skin (Figure 1). [1] A true TAWH must also be absent of preexisting hernia at the injury site, have evidence of abdominal wall injury at presentation, and demonstrate immediate or delayed hernia development with or without presence of a peritoneal sac. [1-3] Classified into three major types on the basis of their mechanism of injury and hernia size, Type I TAWHs are small defects caused by direct blow such as handlebar injuries, commonly known as handlebar hernias $(\mathrm{HH})$, type II TAWHs are larger and caused by high energy mechanisms such as motor vehicle crashes (MVC) or fall from heights, and type III TAWHs are intrabdominal bowel herniation caused by deceleration injuries. [1, 3-4]

The reported incidence in TAWH in children is $1 \%$ but can be up to $85 \%$ due to $\mathrm{HH}$. [3, 5-9] Associated rates of intrabdominal injury vary widely between $25-70 \%$, while some report lower rates in $\mathrm{HH}$ and higher rates in MVC. $[3,5-6,10]$ As a result of the large variation of reported rates of both TAWH and intrabdominal injuries, management remains debated as the growing body of work supports nonoperative management. $[8,11-12]$ We present the largest known retrospective review of children suffering a TAWH from a large Level 1 pediatric trauma center and describe its method of diagnosis, frequency of concurrent intrabdominal injury (CAI) requiring intervention, and treatment. 


\section{METHODS:}

\section{1. Study Design}

A retrospective review of all pediatric patients who suffered from TAWH after blunt abdominal trauma over an eleven-year period (2009-2019) was completed at a Level 1 Pediatric Trauma center. Patients were identified through the trauma registry from a single institution (Riley Hospital for Children at IU Health, Indianapolis, Indiana). Institutional Review Board (IRB) approval as well as a waiver of consent was obtained from Indiana University. Patient demographics, injury severity score (ISS), mechanism of injury (MOI), radiologic and operative findings, as well as clinical course were recorded. Patients who suffered penetrating trauma, those with a traumatic abdominal wall hernia involving skin penetration, and those solely with a traumatic diaphragmatic hernia were excluded.

\section{2. Data Extraction and Analysis}

The electronic medical record of each patient with a TAWH was reviewed to determine the relevant preoperative findings, indications for the operating room (OR), operative findings and management, and outcomes. All preoperative data was extracted from the work up at initial presentation and analysis of these findings excluded those patients in whom this information was not available. CT scan findings were based on preoperative interpretation by the radiologist and/or surgeon when available in the medical records and did not account for addended or changed impressions following an over-read of the scan after OR management had taken place. Operative findings from immediate and delayed procedures were reviewed. Follow-up was evaluated from outpatient clinic records. When available, CT scan findings and operative findings were compared to determine the rate of significant concurrent intrabdominal injury 
(CAI). CAI was defined as major gastrointestinal (GI) or genitourinary (GU) injury that required operative attention and management at index exploration, excluding those injuries managed nonoperatively such as a solid organ injury.

\section{3. Statistics}

Descriptive statistics were utilized throughout. Continuous data were reported as the mean $+/$ - one standard deviation. Categorical data were reported as frequencies and percentages. 
3. RESULTS:

\section{1. Study Population}

Over the 11-year period, 9370 patients suffered blunt abdominal trauma. Fifteen patients were initially found with intrabdominal fascial disruptions. After exclusion of two penetrating injuries, one full thickness defect, and one traumatic diaphragmatic hernia, 11 patients were identified with an incidence of $0.1 \%$ (Figure 2). Eight (73\%) patients were male. No child had any history of previous hernias or major abdominal surgical history. All patients except two were hemodynamically normal, had a mean ISS of 16, and mean Glasgow Coma Scale (GCS) of 13 on arrival. Three patients had abnormal laboratory values with elevated transaminases on initial trauma work up. Eight patients had other organ system injuries: five with orthopedic injuries, five with vascular injuries, four with intrathoracic injury, four with neurologic or spine injuries, and four with skin and/or soft tissue injuries.

\section{2. TAWH Mechanism of Injury, Type, and Location}

Six patients (55\%) presented after a high speed MVC, two (18\%) after bicycle handlebar injury, one (9\%) after impalement by a pole, and two (18\%) were dragged under a large vehicle (Table 1). Over half of the patients had a type II TAWH (Table 1).

Twelve hernias were found amongst the 11 patients with TAWH (Table 1). Four patients had hernias involving more than one region. The anterior right lower quadrant (RLQ) was the most common location for a TAWH followed by posterolateral locations (Table 1). All cases that were not Type II hernias occurred in the RLQ, except for one patient struck by a pole who had both a RLQ hernia as well as a left posterolateral hernia.

\section{3. TAWH and Concurrent Intrabdominal Injuries Diagnoses}


Every patient presenting with a TAWH demonstrated positive physical examination findings such as bruising or tenderness. However, the detection rate of the hernia itself on examination was low (Table 2). On CT imaging, two TAWHs were not detected on initial CT interpretation but later confirmed on retrospective review. Every patient demonstrated an intrabdominal CT finding, with four patients' CT scans showing intrabdominal injuries needing surgical intervention.

Ten of eleven patients were taken to the OR within the first 12 hours of presentation to the hospital. Nine of these ten patients proceeded to the OR within 0 to 3 hours of hospital presentation and one patient underwent OR exploration at 9 hours; this timing was primarily driven by hard indications for operative exploration in a blunt trauma patient such as hemodynamic instability or peritonitis, as well as clinical suspicion of associated concomitant injury (Table 2). The one remaining patient who did not proceed to the OR required nephrostomy tube placement by interventional radiology (IR) for a ureteral transection; an unsuccessful attempt was first made within the first hour of hospital arrival and reattempted with success at 22 hours.

In sum, there were six patients with associated intrabdominal injuries found intraoperatively, and seven total who required major intervention in IR or OR on admission. Preoperative CT scans suspecting concurrent intrabdominal injury (CAI) only had 20\% sensitivity and 50\% specificity (Figure 2).

\section{4. TAWH with Confirmed Concurrent Intrabdominal Injuries}

Compared to the entire group of patients with TAWH ( $\mathrm{n}=11)$, patients with TAWH and CAI ( $\mathrm{n}=6)$ who required OR had a higher mean ISS (22.5 versus 15.9$)$ and were more likely to have hernias involving the RLQ (71.4\% versus $58.3 \%)$. Otherwise this group had similar 
demographics, presenting hemodynamics, physical exam findings, and CT scan findings (Tables $1-2)$.

\section{5. Operative Management and Outcomes}

Ten patients proceeded to the OR and one patient required IR intervention within 12 hours of admission. Three patients required damage control operations; two of these patients had a delayed hernia repair during index admission, and one of these remains unrepaired (Table 3). Three TAWH were not repaired on index admission; one has since resolved on follow up imaging, and the remaining two remain present but asymptomatic on outpatient follow up (Table 3). All repaired hernias were approached in an open fashion and were approximated primarily without mesh. Patients with TAWH and CAI were less likely to have their hernia repaired on

index exploration. Overall, there were no complications, hernia recurrences after repair, or deaths. 


\section{DISCUSSION:}

TAWH are rare, and available literature is primarily based on case reports and small case series describing these injuries. This review provides the largest series from a single pediatric Level 1 trauma institution. Use of the trauma registry database allows a complete capture of blunt abdominal injuries and provides a TAWH incidence of $0.1 \%$ in patients admitted after blunt abdominal trauma. This is lower than the current reported rate of $1 \%$ and may reflect a more complete collection of patients. [5-7] While up to $85 \%$ of TAWH in children are thought to be due to $\mathrm{HH}$, our series showed that $55 \%$ were due to MVCs while only $18 \%$ were from $\mathrm{HH}$. [3, 8-9] These differences might be attributed to the larger number of blunt abdominal trauma patients seen by our large Level 1 children's trauma hospital serving an entire state as a referral center for urban and rural populations.

For optimal management of TAWH, rates of CAI must also be considered. Overall rates have been reported between $25-70 \%$, which also accounts for injuries managed purely through nonoperative means. Some series show different rates based on mechanism of injury with $8 \%$ injury rate in $\mathrm{HH}$ and $25 \%$ injury rate in MVCs. [3, 6, 8, 10, 13] Given the blunt trauma to a localized area that can transfer significant enough force to the abdominal wall to create a traumatic hernia, clinicians must maintain high suspicion for the coexistence of common intrabdominal injuries, such as to bowel, mesentery and pancreas, as these may not be easily apparent on CT scans and can manifest in a delayed fashion. [3, 14-15] Our review revealed 64\% 
of patients had a concurrent intrabdominal injury, most commonly bowel and mesenteric defects, all of which required major intervention, with one undergoing major interventional procedures and six undergoing operative intervention. The results also had nearly equal distribution between CAI rates and the mechanism of injury, which more closely mimics TAWH and CAI rates observed in adults. [3, 16-19]

Given the rarity of TAWH, accurate diagnosis of both the abdominal wall hernia and associated injuries is challenging. CT scan has gained popularity as the diagnostic modality of choice due to its perceived ability to detect the hernia as well as associated intrabdominal injury. $[3,20]$ Our series found that CT scans detected most TAWHs where physical examination fell short, yet operative intervention was more likely dependent on clinical factors, such as worsening examination despite otherwise nonspecific or negative CT scan findings. This supports the need for keeping a high index of suspicion for injury requiring operative intervention, with serial examination and monitoring with special attention to concern for evolution of gastrointestinal tract injury. [21-22]

While the presence of this TAWH was highly associated with a concurrent intrabdominal injury needing intervention, we found that CT scans were poor predictors of CAI despite the presence of TAWH and nonspecific findings such as abdominal free fluid. With the combination of poor predictability and high incidence of CAI, we advocate for early exploration, within the first 12 to 24 hours of arrival, in children suffering TAWH in order to evaluate for a CAI that may warrant urgent operative attention while also managing the hernia. In our series, all patients proceeded to undergo a major intervention within the first 12 hours of their arrival. We believe that patients found to have a TAWH on workup can proceed with exploration in an urgent to emergent basis, where those trauma patients who demonstrate hard signs for exploration at 
presentation such as hemodynamic instability despite appropriate resuscitation or peritonitis proceed to the OR emergently, while those without these initial findings can be managed in an urgent manner within the first 12 to 24 hours of arrival after initial stabilization and resuscitation have allowed for a more optimal operative setting and candidate.

Operative management, timing of hernia repair and type of repair should be on a case by case basis as they depend on the patient's stability and intrabdominal findings, including degree of contamination and need for temporary abdominal closure. In cases of hemodynamic stability where exploratory laparotomy may not be necessary or where diagnosis is unclear, laparoscopy can be considered. $[3,10]$ Use of laparoscopy as an adjunct to both the diagnosis of TAWH in situations where the hernia would otherwise be missed, as well as to explore abdominal contents for associated injury, is one consideration in management of such patients following an otherwise unremarkable or nonspecific CT scan. [1, 3, 10, 23]

Hernia repair itself will first depend on intrabdominal findings, and when feasible, debridement of nonviable tissue and primary tension-free repair with nonabsorbable suture is recommended upon TAWH diagnosis to prevent future complications such as bowel obstruction, incarceration, or strangulation. [3, 6] Whereas the use of prosthetic material is commonplace in adults, use of mesh in pediatrics is rarely needed and reserved for large defects where a tensionfree primary repair is not possible. $[3,6,19]$ In our series, those patients who did have CAI were less likely to have their TAWH repaired immediately and had no major differences in outcomes such as hernia complications or symptoms. This may have been due to concern for development of compartment syndrome or need for second look procedures in the trauma setting, although that could not be assessed from the operative records. This suggests that perhaps early 
nonoperative management of the hernia itself may be acceptable once CAI injury and any other complicating factor has been ruled out and/or managed. $[8,11-12]$

While this study provides the largest case series of children suffering TAWH to date, there are several limitations. Despite the large volume of blunt abdominal trauma seen at our pediatric trauma center, only 11 patients were found with a TAWH over the 11-year study period. The small patient cohort from a single institution along with the retrospective nature of the study introduces bias and unmeasured confounders that limit the generalizability of the conclusions. Furthermore, there was no set protocol for determining candidates for exploration and its optimal timing, and our retrospective findings reflect surgeon-dependent decision-making for choosing exploration. 


\section{CONCLUSION:}

This retrospective review from a large Level 1 pediatric trauma center provides the largest single series of children with traumatic abdominal wall hernia and confirms the rare incidence of this diagnosis. In our series, these hernias are most commonly due to motor vehicle accidents followed by handlebar injuries and are associated with high rates of concurrent intrabdominal injuries that necessitate operative management. While the TAWH is detected readily on CT scan, there is a low sensitivity and specificity in detecting these concurrent injuries. The presence of a TAWH should key in the clinician to the coexistence of commonly missed diagnoses, such as injuries to bowel, mesentery, and pancreas. Therefore, a high index of suspicion for associated intrabdominal injury should be maintained, with a low threshold for operative exploration and careful ongoing monitoring for evidence of delayed progression of additional injuries.

Acknowledgements: Indiana University School of Medicine

Funding: This research did not receive any specific grant from funding agencies in the public, commercial, or not-for-profit sectors.

Declarations of interest: None 


\section{REFERENCES:}

[1] Goliath J, Mittal V, McDonough J. Traumatic handlebar hernia: a rare abdominal wall hernia. J Pediatr Surg. 2004;39:e20-22. https://doi.org/10.1016/j.jpedsurg.2004.06.039

[2] Mancel B, Aslam A. Traumatic abdominal wall hernia: an unusual bicycle handlebar injury. Pediatr Surg Int. 2003;19:746-747. https://doi.org/10.1007/s00383-003-1064-8

[3] Talutis SD, Muensterer OJ, Pandya S, McBride W, Stringel G. Laparoscopic-assisted management of traumatic abdominal wall hernias in children: case series and a review of the literature. J Pediatr Surg. 2015;50:456-461. https://doi.org/10.1016/j.jpedsurg.2014.10.020

[4] Wood RJ, Ney AL, Bubrick MP. Traumatic abdominal hernia: A case report and review of the literature. Am Surg. 1988;54:648-651.

[5] Moremen JR, Nakayama DK, Ashley DW, Astin M, Nolan TL. Traumatic disruption of the abdominal wall: Lap-belt injuries in children. J Pediatr Surg. 2013;48:e21-24. https://doi.org/10.1016/j.jpedsurg.2013.02.039

[6] Rinaldi VE, Bertozzi M, Magrini E, Riccioni S, Di Cara G, Appignani A. Traumatic Abdominal Wall Hernia in Children by Handlebar Injury: When to Suspect, Scan, and Call the Surgeon. Pediatr Emerg Care. 2017. https://doi.org/10.1097/PEC.0000000000001153

[7] Theodorou CM, Galganski LA, Stark RA. Traumatic posterolateral abdominal wall hernia in a pediatric patient. J Pediat Surg Case Reports. 2019;43:23-25. https://doi.org/10.1016/j.epsc.2019.01.013

[8] Volpe A, Virgone C, Gamba P. Successful conservative management of handlebar hernia in children. Pediatr Int. 2016;59: 105-106. https://doi.org/10.1111/ped.13110

[9] Pickett D, Geiger J, Leskovan JJ, Moore J, Stausmire JM. Lap-Belt-Induced Pediatric Blunt Traumatic Abdominal Wall Hernia. Am Surg. 2017;83(6):e189-191.

[10] Rathore A, Joyce Simpson B, Diefenbach KA. Traumatic abdominal wall hernias: an emerging trend in handlebar injuries. J Pediatr Surg. 2012;47:1410-1413. https://doi.org/10.1016/j.jpedsurg.2012.02.003

[11] Litton K, Izzidien AY, Hussien O, Vali A. Conservative management of a traumatic abdominal wall hernia after a bicycle handlebar injury (case report and literature review). $\mathrm{J}$ Pediatr Surg. 2008; 43:e31-32. https://doi.org/10.1016/j.jpedsurg.2007.12.059

[12] Matsuo S, Okada S, Matsumata T. Successful conservative treatment of a bicyclehandlebar hernia: report of a case. Surg Today. 2007;37(4):349-51. https://doi.org/10.1007/s00595-006-3375-6

[13] Mitchell PJ, Green M, Ramesh AN. Handlebar hernia in children. Emerg Med J. 2011;28(5):439-440. https://doi.org/10.1136/emj.2009.089870

[14] Nadler EP, Patoka DA, Shultz BL, Morrison KE, Ford HR, Gaines BA. The High Morbidity Associated with Handlebar Injuries in Children. J Trauma. 2005;58(6):11711174. https://doi.org/10.1097/01.ta.0000170107.21534.7a

[15] Vandewalle RJ, Barker SJ, Raymond JL, Brown BP, Rouse TM. Pediatric Handlebar Injuries More Than Meets the Abdomen. Pediatr Emerg Care. 2019;1-7. https://doi.org/10.1097/PEC.0000000000001690

[16] Netto FA, Hamilton P, Rizoli SB, Nascimento B, Brenneman FD, Tien H, Tremblay LN. Traumatic Abdominal Wall Hernia: Epidemiology and Clinical Implications. J Trauma. 2006;61(5):1058 -1061. https://doi.org/10.1097/01.ta.0000240450.12424.59 
[17] Honaker D, Green J. Blunt traumatic abdominal wall hernias: Associated injuries and optimal timing and method of repair. J Trauma Acute Care Surg. 2014;77(5): 701-704. https://doi.org/10.1097/TA.0000000000000431

[18] Coleman JJ, Fitz EK, Zarzaur BL, Steenburg SD, Brewer BL, Reed L, Feliciano DV. Traumatic abdominal wall hernias: Location matters. J Trauma Acute Care Surg. 2016;80(3): 390-397. https://doi.org/10.1097/TA.0000000000000946

[19] Suhardja TS, Atalla MA, Rozen WM. Complete Abdominal Wall Disruption With Herniation Following Blunt Injury: Case Report and Review of the Literature. Int Surg. 2015;100:531-539. https://doi.org/10.9738/INTSURG-D-14-00079.1

[20] Ciftci AO, Salman B, Turken A, Senocak ME. Acute Blunt Traumatic Abdominal Wall Hernia. J Pediatr Surg. 1997. 32(12):1732-1734. https://doi.org/10.1016/s00223468(97)90518-2

[21] Drucker NA, McDuffie L, Groh E, Hackworth J, Bell TM, Markel TA. Physical Examination is the Best Predictor of the Need for Abdominal Surgery in Children Following Motor Vehicle Collision. J Emerg Med. 2018;54(1):1-7. https://doi.org/10.1016/j.jemermed.2017.08.008

[22] Paris C, Brindamour M, Ouimet A, St-Vil D. Predictive indicators for bowel injury in pediatric patients who present with a positive seat belt sign after motor vehicle collision. J Pediatr Surg. 2010;45:921-924. https://doi.org/10.1016/j.jpedsurg.2010.02.023

[23] Iinuma Y, Yamazaki W, Hirose Y, Kinoshita H, Kumagai K, Tanaka T, Miyajima M, Nitta K, Naitoh S, Kobayashi K. A case of a traumatic abdominal wall hernia that could not be identified until exploratory laparoscopy was performed. Pediatr Surg Int. 2005;21:54-57. https://doi.org/ 10.1007/s00383-004-1264-x 
Table 1: TAWH Details

\begin{tabular}{lll}
\hline & $\begin{array}{l}\text { All TAWH (n=11) } \\
\text { n }(\%)\end{array}$ & $\begin{array}{l}\text { TAWH+CAI (n=6) } \\
\mathbf{n}(\%)\end{array}$ \\
\hline \hline Age & $10.0 \pm 2.1$ & $10.3 \pm 2.5$ \\
ISS & $15.9 \pm 13.9$ & $22.5 \pm 14.4$ \\
Mechanism of Injury & 11 & 6 \\
$\quad$ Handlebar & $2(18.2)$ & $1(16.7)$ \\
$\quad$ High speed MVC & $6(54.5)$ & $3(50.0)$ \\
Other & $3(27.3)$ & $2(33.3)$ \\
TAWH Type & 11 & 6 \\
Type I & $3(27.3)$ & $2(33.3)$ \\
$\quad$ Type II & $6(54.5)$ & $3(50.0)$ \\
Type III & $0(0)$ & $0(0)$ \\
Other & $2(18.2)$ & $1(16.7)$ \\
TAWH Location & 12 & 7 \\
RUQ or LUQ & $0(0)$ & $0(0)$ \\
RLQ & $7(58.3)$ & $5(71.4)$ \\
LLQ & $3(25)$ & $1(14.3)$ \\
Rectus Abdominis & $3(25)$ & $2(28.6)$ \\
Posterolateral- R & $3(25)$ & $2(28.6)$ \\
Posterolateral- L & $3(25)$ & $1(14.3)$ \\
\hline
\end{tabular}

${ }^{+}$One child had two distinct hernias

${ }^{\text {F}}$ Four children had one or more areas involved due to large hernia size 
Table 2: Diagnosis of TAWH and Concurrent Intrabdominal Injuries

\begin{tabular}{lll}
\hline & $\begin{array}{l}\text { All TAWH (n=11) } \\
\text { n (\%) }\end{array}$ & $\begin{array}{l}\text { TAWH+CAI (n=6) } \\
\text { n (\%) }\end{array}$ \\
\hline Physical Exam Findings & 11 & 6 \\
Hernia palpable/identified & $3(27.3)$ & $2(33.3)$ \\
Abdominal ecchymosis/seatbelt sign & $10(90.9)$ & $5(83.3)$ \\
Tenderness/peritoneal signs & $8(72.3)$ & $5(83.3)$ \\
Open wound defect & $1(9.1)$ & $1(16.7)$ \\
CT Imaging Findings & $10^{\wedge}$ & 5 \\
Hernia present & $8(80)$ & $4(80)$ \\
Free fluid & $9(90)$ & $5(100)$ \\
Free air & $1(10)$ & $0(0)$ \\
Bowel injury & $4(40)$ & $2(40)$ \\
Solid organ injury (Spleen, Liver, Kidney) & $3(30)$ & $2(40)$ \\
Vascular Abnormality & $2(20)$ & $1(20)$ \\
OR Indication & $10^{\ddagger}$ & 6 \\
Worsening abdominal exam/peritonitis & $2(20)$ & $2(33.3)$ \\
Hemodynamic instability & $1(20)$ & $1(16.7)$ \\
Concern for bleeding/vascular injury & $3(30)$ & $2(33.3)$ \\
Concern for bowel injury & $3(30)$ & $2(33.3)$ \\
Free fluid on imaging & $1(10)$ & $1(16.7)$ \\
TAWH containing bowel* & $3(30)$ & $1(16.7)$ \\
Operative Findings requiring intervention & $7 / 11(63.6)$ & $6 / 11(54.5)$ \\
Bowel injury & 5 & 5 \\
Mesenteric injury & 4 & 4 \\
Urologic injury & 2 & 1
\end{tabular}

${ }^{1} 1$ patient without preoperative CT

${ }^{\ddagger} 1$ patient did not proceed to OR within first 12 hours of admission 
Table 3: Surgical Management and Hernia Repair

\begin{tabular}{cll}
\hline & $\begin{array}{l}\text { All TAWH }(\mathbf{n}=\mathbf{1 1}) \\
\mathbf{n}(\mathbf{\%})\end{array}$ & $\begin{array}{l}\text { TAWH+CAI (n=6) } \\
\mathbf{n}(\mathbf{\%})\end{array}$ \\
\hline \hline Trauma Operative approach & $10^{\ddagger}$ & 6 \\
Laparoscopy & $3(30)$ & $1(16.7)$ \\
Laparotomy & $7(70)$ & $5(83.3)$ \\
Hernia management & 11 & 6 \\
Immediate repair & $5(45.4)$ & $2(33.3)$ \\
Delayed repair & $3(27.3)$ & $2(33.3)$ \\
Nonoperative & $3^{\#}(27.3)$ & $2^{\#}(33.3)$ \\
\hline
\end{tabular}

¥1 patient did not proceed to OR within first 12 hours of admission

${ }^{\#} 1$ found to be resolved on follow up imaging 
Highlights

- Traumatic abdominal wall hernia is a rare sequela of blunt abdominal trauma in kids

- Kids with traumatic abdominal wall hernias often have a concurrent abdominal injury

- CT scans have poor detection rates for these concurrent intrabdominal injuries

- High suspicion for concurrent internal injury should be kept with traumatic hernias

- Early exploration should be considered with traumatic abdominal wall hernias 

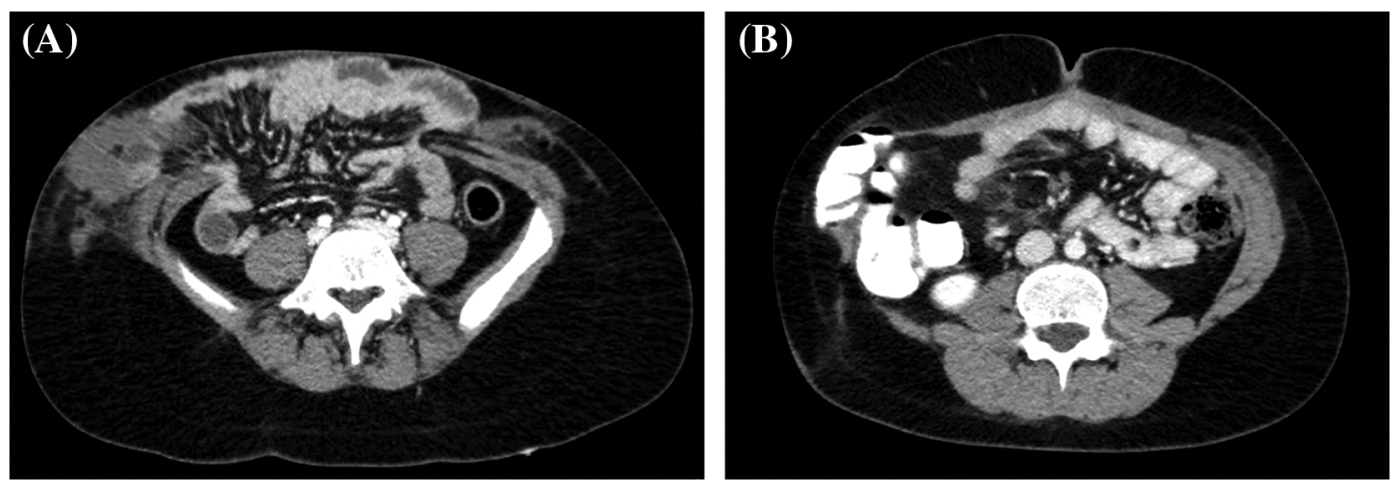

Figure 1 
9370 children with blunt abdominal trauma

4 Exclusions 15 Traumatic fascial disruption

2 penetrating

1 full thickness defect

1 traumatic $\mathrm{DH}$

11 TAWH

1 to OR due

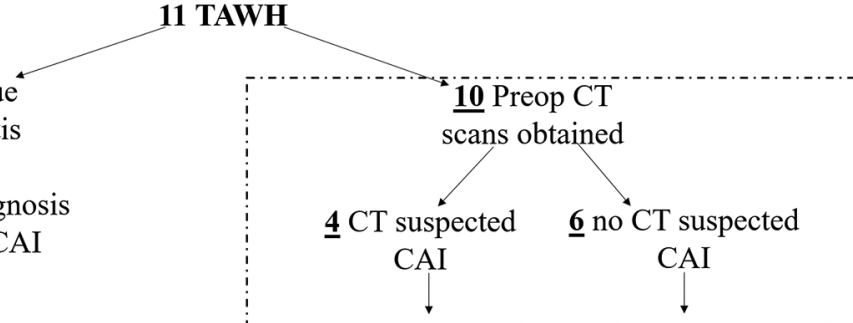

- 1 confirmed CAI intraop

- 4 intraop diagnosis

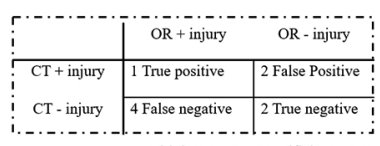

- I IR intervention Sensitivity $=20 \% \quad$ Specificity $=50 \%$ for GU injury

Figure 2 